\title{
Ultrasound-guided carpal tunnel injections
}

\author{
Tilen Tumpaj', Vesna Potocnik Tumpaj², Domenico Albano³, Ziga Snoj²,4 \\ ${ }^{1}$ Department of Anaesthesiology and Intensive Care Medicine, University Medical Centre Ljubljana, Slovenia \\ ${ }^{2}$ Institute of Radiology, University Medical Centre Ljubljana, Slovenia \\ ${ }^{3}$ Istituto Ortopedico Galeazzi, IRCCS Milano “Galeazzi”, Unit of Diagnostic and Interventional Radiology Milan, Italy \\ ${ }^{4}$ Faculty of Medicine, University of Ljubljana, Ljubljana, Slovenia
}

Radiol Oncol 2022; 56(1): 14-22.

Received 10 Avgust 2021

Accepted 15 December 2021

Correspondence to: Tilen Tumpaj, M.D., Department of Anaesthesiology and Intensive Care Medicine, University Medical Centre Ljubljana, Zaloška 7, SI-1000 Ljubljana, Slovenia. E-mail: tilen.tumpaj@gmail.com

Disclosure: No potential conflicts of interest were disclosed.

This is an open access article under the CC BY-NC-ND license (http://creativecommons.org/licenses/by-nc-nd/4.0/).

Background. Carpal tunnel syndrome (CTS), one of the most common entrapment neuropathies, can, in fact, be considered as a socio-economic issue that reduces work productivity, increases disability, and requires prolonged rehabilitation. The imaging modality of choice in CTS imaging is the ultrasound (US), as several morphological parameters can be used in CTS diagnosis and follow-Up. In recent years, US-guided CTS injection therapy has become an established treatment option for mild to moderate CTS. The authors of this review performed a literature search that revealed several differences in US-guided carpal tunnel injection in an attempt to unify individual stages of CTS injections protocol for future guidance: patient preparation, injection approach, needle positioning, injected medications, and injectate volume. The three approaches to carpal tunnel injections described in the literature, that is, the ulnar, radial, and longitudinal, can be implemented with single or multiple deposits and different injection volumes. Medications used for injections are corticosteroids, local anaesthetics, dextrose, saline, platelet-rich plasma, and progesterone.

Conclusions. Although no consensus has yet been reached as to which protocol should be used, the ulnar approach with a single deposit injected in large volumes should be considered as the first choice, while dextrose should be the first-line medication option. Furthermore, as terminological differences make it difficult to draw a uniform comparison the presented steps for US-guided carpal tunnel injection might serve as a guideline for future studies.

Key words: carpal tunnel syndrome; ultrasound-guided injections; injection approach; needle positioning; corticosteroid; local anaesthetics

\section{Introduction}

Carpal tunnel syndrome (CTS), one of the most common entrapment neuropathies, can, in fact, be considered as a socio-economic issue that reduces work productivity, increases disability, and requires prolonged rehabilitation. ${ }^{1}$ The diagnostic workup of CTS must include a comprehensive evaluation including clinical findings, nerve conduction studies, and ultrasound imaging (US) ${ }^{2,3}$, which represents the modality of choice due to the superficial course of the median nerve $(\mathrm{MN}))^{3,4}$ Nerve US examination is performed with high- frequency probes that provide a detailed depiction of nerve echotexture and fascicles. ${ }^{5,6}$ US-based morphological parameters for detecting CTS are an increased cross-section area (CSA) of the MN at the carpal tunnel inlet or outlet, flattening of the $\mathrm{MN}$, and bowing of the transverse carpal ligament (TCL) at the level of the carpal tunnel outlet.-9 Elastography has been proven as a useful adjunct US method in CTS evaluation. ${ }^{10}$

Therapeutic recommendations for CTS depend on disease severity and may include anything from a conservative approach to surgical intervention. ${ }^{2,3}$ US-guided injections have become increasingly 
important in the treatment of mild and moderate $\mathrm{CTS}^{2,3}$ and, as landmark-guided injections have been proven to be less effective and cause more complications, US imaging has become the foundation of therapeutic recommendations., ${ }^{2,3}$ US-guided carpal tunnel injections are the most effective minimally invasive treatment method with minimum side effects that can remarkably improve the symptoms and functional status. ${ }^{11}$ The authors of this review performed a literature search that revealed several differences in US-guided carpal tunnel injections and summarized the differences in an attempt to unify individual stages of CTS injections protocol for future guidance.

\section{Carpal tunnel injections}

\section{Literature search}

For our narrative review paper, we conducted an Ovid MEDLINE and PubMed search in which we included the papers published from 2002, when the first paper on US-guided injection was published ${ }^{12}$, to 2021 with the keywords »carpal tunnel injection « and »ultrasound-guided carpal tunnel injection«. The search results included case-control studies, systematic reviews, and meta-analyses that contained data on US-guided carpal tunnel injections. References in these papers were carefully reviewed and were included in our review if they met our criteria (Figure 1). After we reviewed the papers, we used the differences between them to define the steps of US-guided carpal tunnel injections: patient preparation, approaches, needle positioning, injected medications and injected volumes.

\section{Patient preparation}

The patient can be either seated or lying supine. ${ }^{13}$ When seated, the elbow is resting on the examination table in a $90^{\circ}$ flexion position. ${ }^{14}$ When lying supine, the arm is abducted to $90^{\circ}$ with the elbow extended..$^{11,15,16}$ In both positions the forearm is supinated and the wrist is in a $15-35^{\circ}$ dorsiflexion position. ${ }^{11,15,16}$ Careful US examination is important for injection planning. The wrist is examined with a linear probe along the carpal tunnel and special attention is given to the MN anatomy (size, position, aberrant variants) and the course of blood vessels. ${ }^{13,17,18}$

Authors reported two different procedures for disinfecting the skin 11,16,17,19: some clean the puncture area with an alcohol swab and use a sterile ultrasound gel $^{11}$, while others perform surgical disinfection and use a sterile probe sleeve. ${ }^{16,17,19}$ Even though there is no clear consensus on patient preparation, in recent years some authors (e.g. Guo, Green, Chianca, etc.) have reported that surgical disinfection of the area can help avoid infections of the puncture site or the deep tissues ${ }^{16,17,19}$, which can also be avoided if a sleeve is used for the US probe. A few authors have reportedly used a shortacting local anaesthetic to numb the dermal and subdermal area before performing US-guided carpal tunnel injection ${ }^{15,20}$, but in the majority of cases, the local anaesthetic is added to the injected mixture. ${ }^{21-23}$ Needles of different sizes (22 to 30 Gauges) have been used in US-guided carpal tunnel injections. ${ }^{11,14-16,24}$ Needles with a small diameter cause less pain upon insertion and are less likely to cause nerve damage during the procedure, but are not

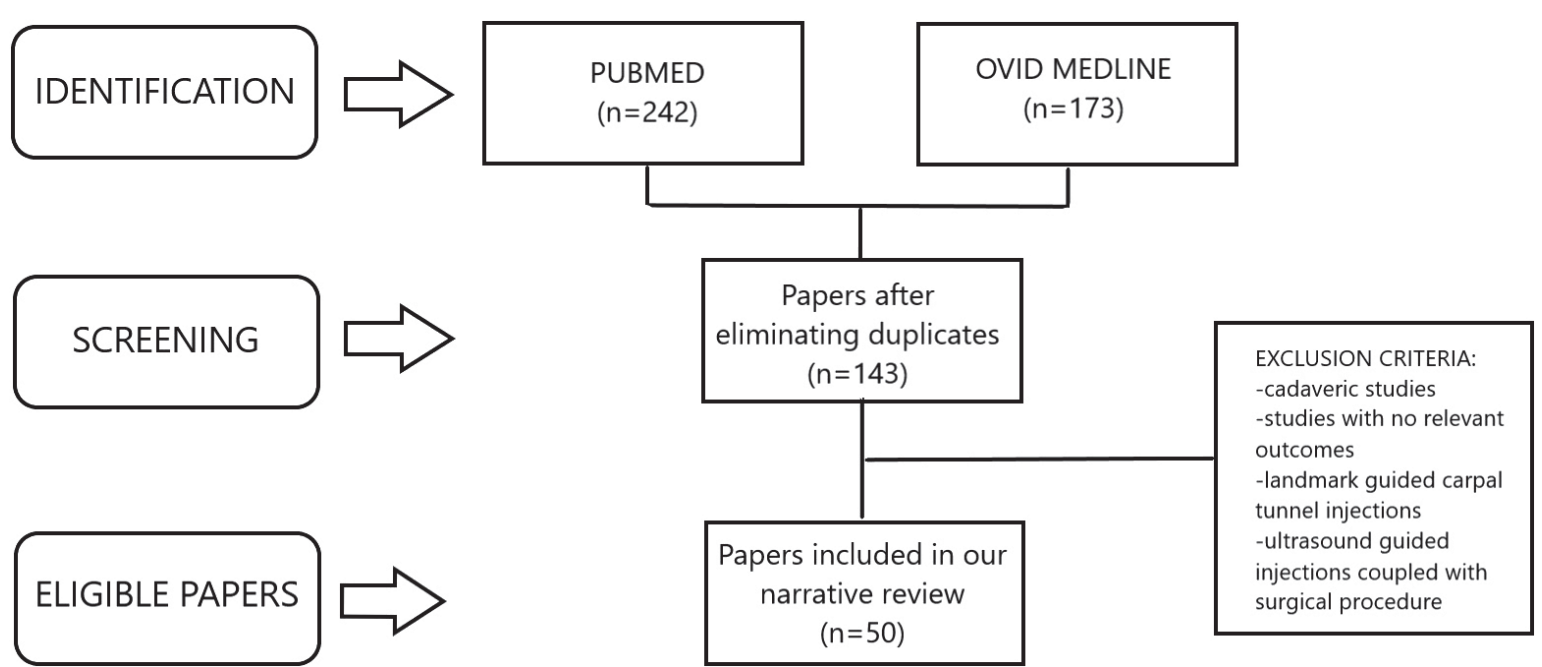

FIGURE 1. Depicting a flowchart of paper search and selection with exclusion criteria. 


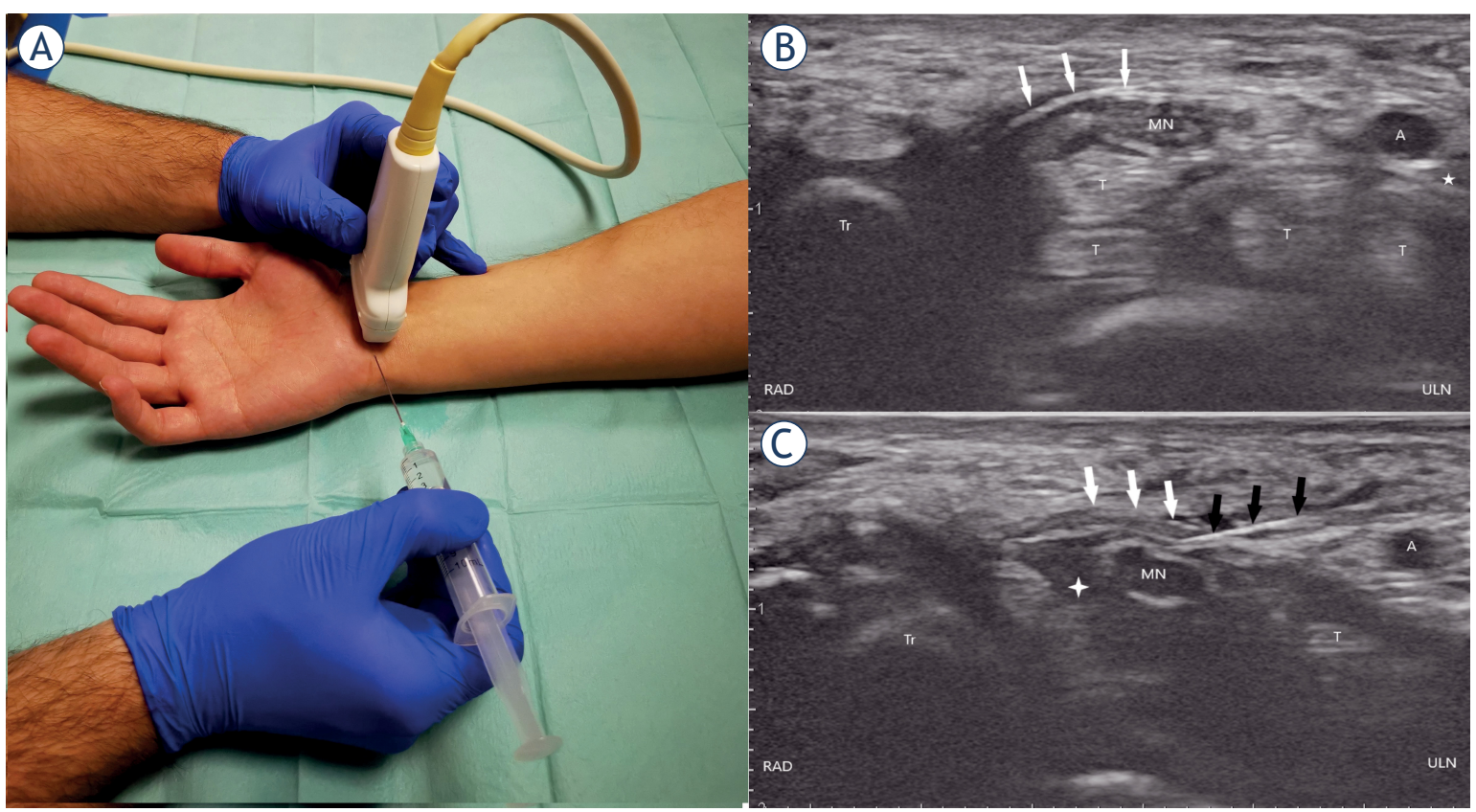

FIGURE 2. (A), (B), and (C) showing the ulnar approach. (A) wrist and needle positioning for carpal tunnel injection, (B) ultrasound anatomy of the carpal tunnel shown in the short axis, (C) penetrating transverse carpal ligament positioning the needle tip above the median nerve. Ultrasound of the carpal tunnel after the needle penetrates the transverse carpal ligament. Comparing $B$ and $\mathrm{C}$ note the expansion of the perineural space marked with a white cross.

$\mathrm{A}=$ ulnar artery; black arrows = needle; $M N=$ median nerve; $R A D=$ radial $; \mathrm{T}=$ flexor tendons; $\mathrm{Tr}=$ trapezium; ULN = ulnar; white arrows = transverse carpal ligament; white cross = perineural injectate; white star = ulnar nerve

suitable for injecting protein-rich plasma, because they damage the platelets due to the small diameter and platelet size. ${ }^{11,14-16,24,25}$

\section{Approaches}

Three approaches to carpal tunnel injections can be found in the literature and all of them are performed with a linear transducer of varying frequencies (5-17 MHz). 11,13,14,26-29

\section{Ulnar approach}

The transducer is positioned at the distal wrist crease perpendicularly to the course of the MN (Figure 2A). ${ }^{13}$ The probe is then moved ulnarly keeping the $\mathrm{MN}$ in view until the pisiform bone, ulnar nerve, and artery are brought into view. On the ulnar side, the pisiform is seen as a hyperechoic structure and the honeycomb appearance of the ulnar nerve may be differentiated radially to the pulsating ulnar artery (Figure 2B). ${ }^{13}$ The needle is introduced in plane in an ulnar to radial direction, then passes the ulnar nerve and ulnar artery superficially, and punctures the TCL so that the needle tip can be advanced adjacently to the MN (Figure 2C). ${ }^{15,26-28}$

\section{Radial approach}

In the radial approach, the transducer is positioned at the distal wrist crease perpendicularly to the course of the MN (Figure 3A). ${ }^{14}$ The probe is moved radially keeping the $\mathrm{MN}$ in view until the scaphoid and flexor carpi radialis tendon are brought into view. On the radial side, the scaphoid is seen as a hyperechoic structure and the flexor carpi radialis tendon lies inferolateral to the $\mathrm{MN}$ (Figure 3B). The needle is introduced in plane in a radial to ulnar direction, then proceeds above the flexor carpi radialis tendon, and punctures the TCL so that the needle tip can be advanced adjacently to the $\mathrm{MN}$ (Figure 3C). ${ }^{14}$

\section{Longitudinal approach}

In the longitudinal approach, the transducer is positioned parallel to the $\mathrm{MN}$ at the distal wrist so that the $\mathrm{MN}$ is seen along the TCL from the carpal tunnel inlet to the carpal tunnel outlet (Figures 4A,B). 


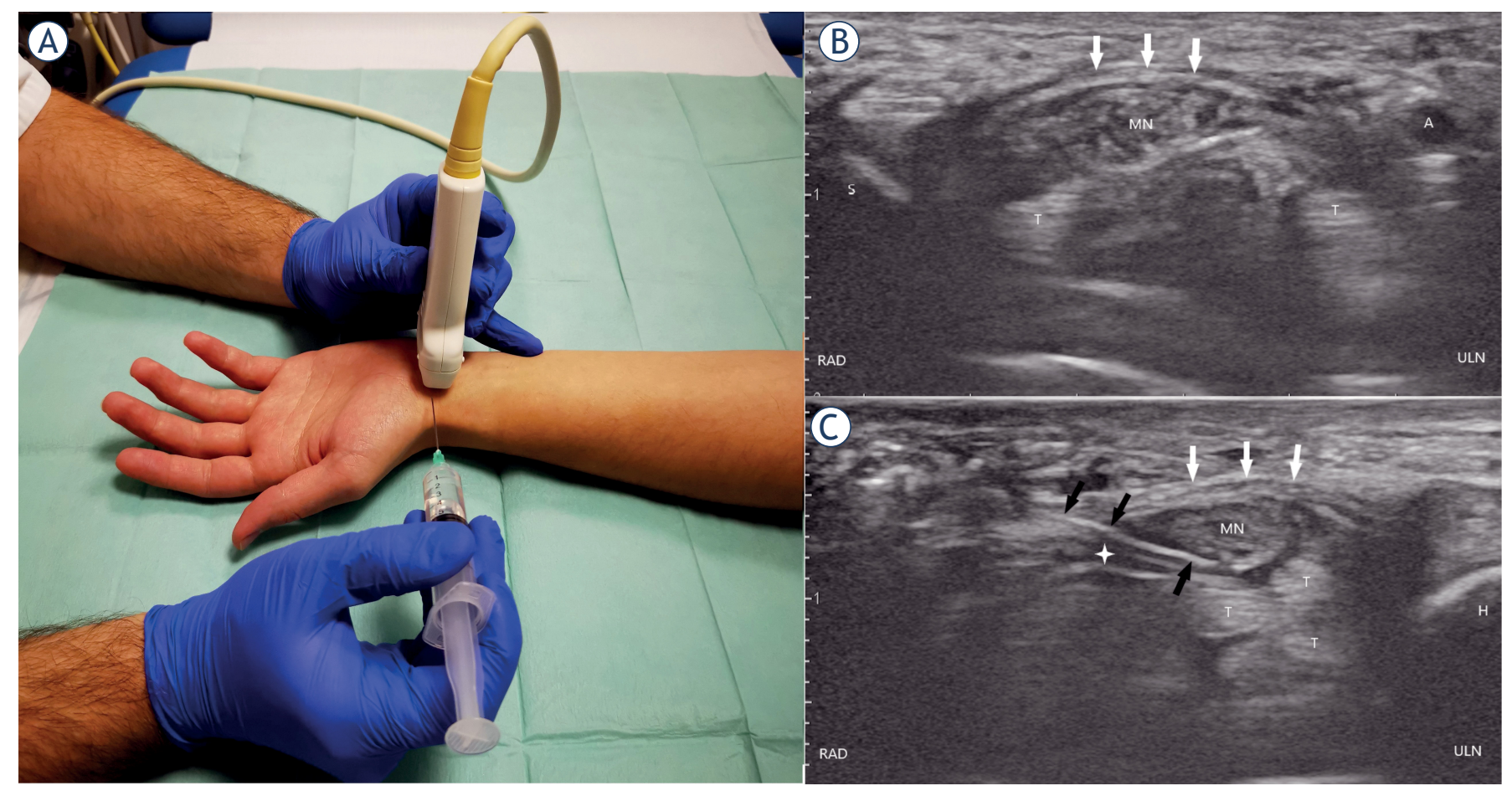

FIGURE 3. (A), (B), and (C) showing the radial approach. (A) wrist and needle positioning for carpal tunnel injection, (B) ultrasound anatomy of the carpal tunnel shown in the short axis, $(C)$ penetrating transverse carpal ligament positioning the needle tip below the median nerve. Ultrasound of the carpal tunnel after the needle penetrates the transverse carpal ligament. Comparing figures $B$ and $C$ note the expansion of the perineural space marked with a white cross.

$\mathrm{A}=\mathrm{ulnar}$ artery; black arrows = needle; $\mathrm{H}=$ hamate; $\mathrm{RAD}=$ radial; $\mathrm{S}=$ scaphoid; $\mathrm{MN}=$ median nerve; $\mathrm{T}=$ flexor tendons; $\mathrm{ULN}=\mathrm{Ulnar} ;$ white arrows = transverse carpal ligament; white cross $=$ perineural injectate

The probe is then moved laterally to the MN approximately $0.5 \mathrm{~cm}$ until the nerve disappears. ${ }^{11}$ Two modifications can be found in the literature, namely the proximal to the distal and the distal to the proximal. ${ }^{11,29}$ In the proximal to distal, the needle is inserted at the distal wrist crease and is advanced distally. ${ }^{29}$ In the distal to proximal, the needle is inserted approximately $2 \mathrm{~cm}$ distally to the distal wrist crease and is advanced proximally. ${ }^{11}$ In both modifications, the needle is introduced in plane and punctures the TCL so that the needle tip can be advanced adjacently to the MN (Figures 4C,D). ${ }^{11,29}$

\section{Needle positioning}

The aim of carpal tunnel injection is to position the needle tip adjacent to the $\mathrm{MN}$ without inducing nerve or vascular injury. When the needle is positioned perineurally, the injection volume can be injected as a single or multiple deposit. In a single deposit, the injection is deposited at a single location $^{11,14,29}$, whereas in a multiple deposit, the needle is repositioned to deposit the injection volume on multiple locations. ${ }^{16}$

\section{Injected medications}

Medications used for carpal tunnel injections are corticosteroids ${ }^{30-35}$, local anaesthetics (LA) ${ }^{35}$, dextrose $^{36,37}$, platelet-rich plasma (PRP) ${ }^{25,38}$, progesterone $^{23,39}$ and saline. ${ }^{40}$ The most widely used medications for carpal tunnel injections are corticoster$\operatorname{oids}^{30-35}$, which ameliorate MN compression due to their anti-inflammatory properties. ${ }^{3}$ Particulate (e.g. methylprednisolone) or nonparticulate (e.g. dexamethasone) corticosteroids can be injected in doses of $40-80 \mathrm{mg} .{ }^{30-35}$ The adverse effects of corticosteroids are rare and range from skin discoloration and irritation at the injection site to neurotoxicity and atrophy of thenar muscles. ${ }^{35}$ Another medication commonly used in the treatment of CTS are LAs ${ }^{35}$, predominantly short-acting LAs (e.g. lidocaine $2 \%$ ) that can be injected as a single compound or in conjunction with corticosteroids. ${ }^{28,35}$ These offer immediate pain relief and may give long-lasting 


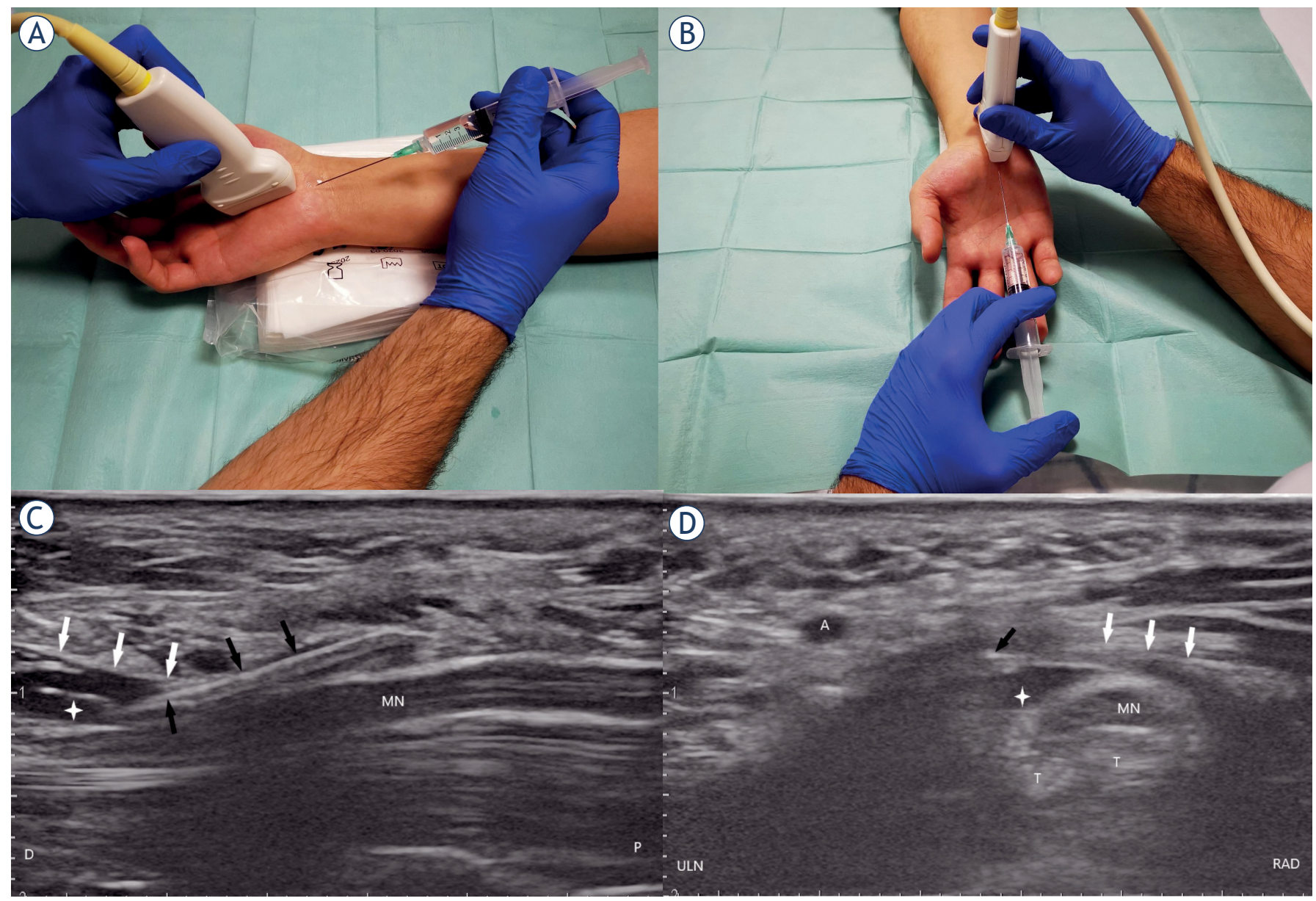

FIGURE 4. (A), (B), (C), and (D) showing the longitudinal approach. (A) Wrist and needle positioning for carpal tunnel injection for the proximal to distal approach, (B) Wrist and needle positioning for carpal tunnel injection for the distal to proximal approach, (C) Ultrasound of the carpal tunnel shown in the long axis after the needle penetrates the transverse carpal ligament and positioning the needle tip parallel to the median nerve, (D) ultrasound of the carpal tunnel shown in the short axis after the needle penetrates the transverse carpal ligament positioning the needle tip parallel to the median nerve. Note in $C$ and $D$ the expanded perineural space marked with a white cross.

$\mathrm{A}=$ ulnar artery; black arrows = needle; $\mathrm{D}=$ distal; $\mathrm{MN}=$ median nerve; $\mathrm{P}=$ proximal $\mathrm{RAD}=$ radial; $\mathrm{T}=$ flexor tendons; $\mathrm{ULN}=\mathrm{ulnar} ;$ white arrows $=$ transverse carpal ligament; white cross $=$ perineural injectate

effects, which are speculated to be caused by the blockage of the sympathetic reflex arc, suppression of nociceptive discharge, blockade of sensitization, and anti-inflammatory effects. ${ }^{35}$ Adverse effects of LAs are rare and range from common allergic reactions to inadvertent intravascular injection, a very serious complication that can lead to seizures and heart conduction blocks. ${ }^{35}$ More recently, dextrose has also been used as a medication. ${ }^{36,37}$ The exact mechanism of action of dextrose is not known, but it is thought that it stimulates an anti-inflammatory response through the inhibition of capsaicin, causing sensitive receptors to prevent the release of substance $\mathrm{P}$ and calcitonin gene-related peptide, both of which are known to cause swelling of the nerve and induce pain. ${ }^{37}$ The main advantage of dextrose is that no serious adverse effects due to biochemical properties were reported. ${ }^{37}$ Saline is widely used either as a single compound in hydrodissection or as a diluting substance for corticosteroids or LAs (Figure 5A,B,C). ${ }^{30-35,40,41}$ There are no serious adverse effects of saline; however, pain upon injection has been reported when no LA is added. $22,28,30,40$ A promising type of injectate is $\mathrm{PRP}^{25,38}$ that triggers a neuroregenerative response by releasing several hormones and growth factors, such as platelet-derived growth factor, transforming growth factor, epidermal growth factor, vascular endothelial growth factor, and insulin-like growth factor-1. These stimulate healing by reducing the 


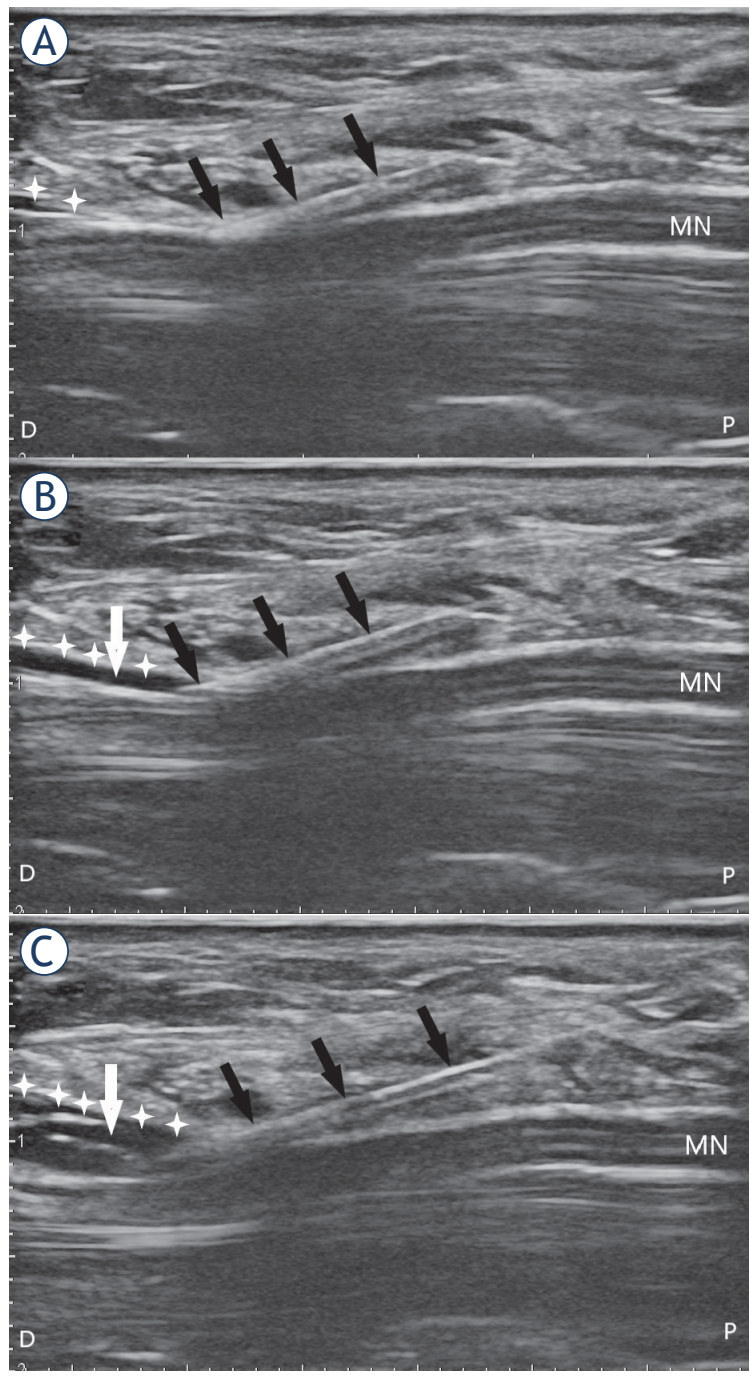

FIGURE 5. (A), (B), and (C) showing the carpal tunnel in the long axis. The effect of injectate volume on perineural space expansion and subsequent hydrodissection. Note the expansion of the perineural space (white arrow) around the median nerve before (A), during (B) and after injecting $6 \mathrm{ml}$ of the injectate and subsequent hydrodissection (C).

black arrows = needle; $\mathrm{D}=$ distal; $\mathrm{MN}=$ median nerve; $\mathrm{P}=$ proximal; white cross $=$ transverse carpal ligament

inflammatory response. ${ }^{38}$ Lastly, progesterone, which has anti-inflammatory and neuroprotective effects on nerves, has also been used recently as an injectate. ${ }^{23,39}$

\section{Injection volume}

There is no consensus on the optimal volume injected into the carpal tunnel and injection volumes anywhere between $1 \mathrm{ml}$ to $10 \mathrm{ml}$ can be found in the literature. ${ }^{30-35,37,40,42-46}$ The injectate can be a manufactured single-compound solution or a multi-compound preparation. The latter is a mixture of active compounds with either saline or LA or both. ${ }^{30-35,40,42-46}$ Corticosteroids are predominantly injected as a multi-compound solution of 1-2 ml corticosteroids with $1-2 \mathrm{ml}$ of LA or 1-2 $\mathrm{ml}$ of saline. ${ }^{28,30-34,45,46}$ LAs are usually added to multi-compound solutions as an anaesthetic during the injection and are rarely injected as a single compound, but when they are, the volume ranges from 0.5 to 4 ml. ${ }^{35,47}$ Saline is mostly used as a mixture substance for other medications as a part of multi-compound solutions. ${ }^{30-37,40,44-46}$ In hydrodissection, saline is used as a single-compound solution of $3-10 \mathrm{ml}$ in volume. ${ }^{40,48}$ Dextrose is injected as a single-compound solution of $3-10 \mathrm{ml}$ in volume. . $^{37,40,48} \mathrm{PRP}$ is injected as a single-compound solution of $1-3 \mathrm{ml}$ in volume. ${ }^{25,38}$ Progesterone is injected as a multicompound solution, a mixture of $0.5 \mathrm{ml}$ of LA and $0.5 \mathrm{ml}$ of progesterone. ${ }^{23,39}$

\section{Discussion}

Even though US-guided injection therapy has become an established treatment option for CTS2, no consensus has yet been achieved on what steps should be taken to achieve the best results. Carpal tunnel injections may be performed with the landmark-guided approach, but several complications have been noted, such as nerve injury, intravascular application of medication, failure to perforate TCL, etc. ${ }^{18}$ When the US is used to guide the injections, the risk of these complications is reduced..$^{18}$

Three approaches to carpal tunnel injections can be found in the literature. The ulnar approach is the most frequently used, as it helps better visualize the carpal tunnel content and thus enables accurate perineural injection by avoiding neurovascular structures. ${ }^{13}$ This approach is also easier to learn in comparison to the other two approaches and provides good needle control. ${ }^{13}$ Reports on radial and longitudinal approaches are scarce. 14,27,29,49 The reviewed authors do not offer any personal perspective on the benefits and difficulties of the radial approach..$^{14}$ Jurbala and Burbank have come to the conclusion that the ulnar approach carries a higher risk of inadvertent penetration of the neurovascular structures because the needle is directed toward and not tangential to the MN. ${ }^{11}$ It has to be noted that scanning in the long axis can be challenging because it is difficult to differentiate between swollen nerve fascicles, muscles, and inflamed tendons. ${ }^{11}$ Only a single study was found 
where different approaches - radial and ulnar, to be precise - were compared to one another, but the results showed no difference in patient outcome or measured US parameters..$^{14}$ Even so, the ulnar approach should be considered as the first choice because it is backed by the largest amount of evidence and is easier to learn than other approaches. ${ }^{13}$

Only a few papers included a detailed report on needle positioning, making it difficult to discern the benefits of different approaches..$^{11,16,29} \mathrm{~A}$ randomized controlled study on US-guided single-deposit injections of corticosteroids showed no difference in patient outcome, electrodiagnostic, and US findings in terms of deposition between the MN and TCL or deposition between the MN and flexor tendons. ${ }^{14}$ The authors emphasized that placing the needle below the MN is technically less demanding and is, therefore, the better option for less experienced practitioners. ${ }^{14} \mathrm{~A}$ study by Nwawka et al. where the spread of US-guided injections was observed in different anatomical positions concluded that a single-deposit injection offers circumferential coverage using injection volumes of $2 \mathrm{ml}$, suggesting that it is unnecessary to place the needle between the MN and TCL at multiple sites along the nerve. ${ }^{49}$ These two studies suggest that a single deposit offers circumferential coverage of the MN. ${ }^{14,49}$ Further studies are warranted to discern the potential superiority of multiple deposit versus single deposit; however, a single deposit should be considered as the first choice as a multiple deposit is harder to perform and has not yet shown any superior benefits.

Another aspect to be taken into consideration in US-guided CTS injection treatment is the choice of medication. Several medications can be used for carpal tunnel injections, of which the most widely utilized are corticosteroids. ${ }^{30-35}$ Although there is no consensus on what type or dose of corticosteroids achieves the best outcome, it is thought that the effect of particulate corticosteroids lasts longer due to the quick uptake of nonparticulate corticosteroids; however, recent studies did not prove the superiority of either type of corticosteroids. ${ }^{30-35}$ Salman Roghani et al. compared the effects of 40 $\mathrm{mg}$ to $80 \mathrm{mg}$ of methylprednisolone for carpal tunnel injections and found no significant differences in patient outcome. ${ }^{33}$ Similarly, Karimzadeh et al. found no significant differences in patient outcome between $40 \mathrm{mg}$ of methylprednisolone and $80 \mathrm{mg}$ triamcinolone, but on the other hand, Habib et al. emphasized that a lower dose could be beneficial due to fewer side effects such as pain upon injection, glucose control after the procedure, and potential neurotoxicity. ${ }^{31,32}$ The majority of US-guided carpal tunnel injections are performed with LAs as part of the multi-compound solution, but these can also be used as a single-compound solution. The effects of LAs as a single compound are poorly researched and only a few studies have been published on this topic. ${ }^{35,47}$ Karadas et al. compared the effects of LAs and corticosteroids in CTS treatment and found no significant difference in patient outcome. ${ }^{47}$ In recent years, dextrose and PRP have also been used in the treatment of CTS. The effects of dextrose as a single-compound solution have been widely researched $36,37,43,50$, but it is still unclear whether they are caused by the release of anti-inflammatory tissue mediators after the injection or better median nerve gliding due to hydrodissection. Comparison between dextrose, saline, and corticosteroids has shown that dextrose is superior in terms of patient outcome..$^{37,43}$ Due to improved patient outcomes, authors have even proposed that dextrose should be a first-line medication option for patients with CTS. ${ }^{37,43}$ Injections of PRP have also given promising results in CTS treatment. In a meta-analysis by Lin et al. PRP was ranked second to dextrose in the terms of clinical effects. ${ }^{43}$ The downside of PRP is the difference in the preparation protocol, which can produce different clinical outcomes due to compositional differences. ${ }^{43}$ There are also the issues of higher costs, more complex organization, and limited availability of machines needed to prepare PRP. ${ }^{43}$ Progesterone has also been proposed as a possible choice of injectate and its effect has been compared to that of corticosteroids due to their similar molecular structure. ${ }^{23,39}$ According to the proposed theory, progesterone receptors are located on the transverse ligament lining cells and wrist synovial tissue. ${ }^{39}$ Bahrami et al. concluded that progesterone is equal to corticosteroids in patient outcomes, while Raeissadat et al. reported that progesterone is equal or even superior to corticosteroids in symptom relief. ${ }^{23,39}$ Several medications are used in clinical practice with no clinical consensus on medication of choice; however, the papers with the highest level of evidence suggest that dextrose should be the first-line medication option. ${ }^{37,43}$

Injection volume also remains a question of debate as only a few studies compared the effects of different volumes. In most studies, relatively low volumes of injectate were used $(1-3 \mathrm{ml}) .{ }^{42}$ In the study by Lin et al., the authors compared different injection volumes of dextrose $(1,2$, and $4 \mathrm{ml})$ and concluded that the injection of $4 \mathrm{ml}$ provided the best outcome. ${ }^{43}$ However, Schrier et al. were unable to prove the superiority of a $5 \mathrm{ml}$ versus $2 \mathrm{ml}$ in- 
jection of corticosteroids and LA. ${ }^{28}$ It is speculated that larger injected volumes $(>5 \mathrm{ml})$ yield better results due to the conjoined effect of hydrodissection and better injection distribution. ${ }^{30,43}$ With hydrodissection, adhesiolysis can be achieved by separating TCL from the MN and enabling normal tendon gliding. ${ }^{28,41}$ A prospective randomized control trial on the effects of hydrodissection showed a significant improvement of the intervention group at a 3-month follow-up in comparison to the control group. ${ }^{37}$ In the intervention group, a multideposit injection was performed to detach the MN from the TCL and separate the MN from the flexor tendons, whereas in the control group saline was injected subcutaneously. ${ }^{37}$ It has been suggested that a cumulative effect of hydrodissection may be expected if injections are repeated. ${ }^{37,40}$ Although only a few studies have been published on this topic, there appears to be a tendency of better outcomes with larger injected volumes.

Some limitations of our review need to be noted. The majority of papers reported a short-term follow-up and only a few reported a follow-up of up to 12 months. Furthermore, all the reviewed papers provided very little information on the optimal protocol of US-guided carpal tunnel injection. Even though we retrieved a large number of papers, only a few of them focused on a specific question related to the proposed steps in USguided carpal tunnel injection. Further studies are required to fully assess the contribution and efficacy of US-guided injection therapy for CTS, and this paper should serve as a reference to determine which study aims are important.

\section{Conclusions}

In recent years, US-guided injection therapy has become an established treatment option in mild to moderate CTS. Although no consensus has yet been reached as to which protocol gives the best results, the ulnar approach with a single deposit should be considered as the first choice and dextrose as the first-line medication option injected in larger volumes. Furthermore, as terminological differences make it difficult to draw a uniform comparison between the reviewed papers, the presented steps of US-guided carpal tunnel injection might serve as a guideline for future studies.

\section{References}

1. Petrover D, Richette P. Treatment of carpal tunnel syndrome: from ultrasonography to ultrasound-guided carpal tunnel release. Joint Bone Spine 2018; 85: 545-52. doi: 10.1016/j.jbspin.2017.11.003

2. Padua L, Coraci D, Erra C, Pazzaglia C, Paolasso I, Loreti C, et al. Carpal tunnel syndrome: clinical features, diagnosis, and management. Lancet Neurol 2016; 15: 1273-84. doi: 10.1016/S1474-4422(16)30231-9

3. Sconfienza LM, Adriaensen M, Albano D, Allen G, Aparisi Gómez MP, Bazzocchi A, et al; Ultrasound and Interventional Subcommittees of the European Society of Musculoskeletal Radiology (ESSR). Clinical indications for image-guided interventional procedures in the musculoskeletal system: a Delphi-based consensus paper from the European Society of Musculoskeletal Radiology (ESSR)-part III, nerves of the upper limb. Eur Radiol 2020; 30: 1498-506. doi: 10.1007/s00330-019-06479-z

4. Sconfienza LM, Albano D, Allen G, Bazzocchi A, Bignotti B, Chianca V, et al. Clinical indications for musculoskeletal ultrasound updated in 2017 by European Society of Musculoskeletal Radiology (ESSR) consensus. Eur Radio/ 2018; 28: 5338-51. doi: 10.1007/s00330-018-5474-3

5. Albano D, Aringhieri G, Messina C, De Flaviis L, Sconfienza LM. Highfrequency and ultra-high frequency ultrasound: musculoskeletal maging up to $70 \mathrm{MHz}$. Semin Musculoskelet Radiol 2020; 24: 125-34. doi: 10.1055/s0039-3401042

6. Snoj Ž, Serša I, Matičič U, Cvetko E, Omejec G. Nerve fascicle depiction at MR microscopy and high-frequency US with anatomic verification. Radiology 2020; 297: 672-4. doi: 10.1148/radiol.2020201910

7. Gonzalez-Suarez CB, Fidel BC, Cabrera JTC, Dela Cruz FC, Gesmundo MVT, Regala CFG, et al. Diagnostic accuracy of ultrasound parameters in carpal tunnel syndrome: additional criteria for diagnosis. J Ultrasound Med 2019; 38: 3043-52. doi: 10.1002/jum.15012

8. Mallouhi A, Pülzl P, Trieb T, Piza H, Bodner G. Predictors of carpal tunnel syndrome: accuracy of gray-scale and color Doppler sonography. AJR Am J Roentgenol 2006; 186: 1240-5. doi: 10.2214/AJR.04.1715

9. Peer $\mathrm{S}$, Gruber $\mathrm{H}$, Loizides A. Sonography of carpal tunnel syndrome: why, when and how. Imag in Med 2012; 4: 287-97. doi: 10.2217/iim.12.25

10. Snoj Ž, Wu CH, Taljanovic MS, Dumić-Čule I, Drakonaki EE, Klauser AS. Ultrasound elastography in musculoskeletal radiology: past, present, and future. Semin Musculoskelet Radiol 2020; 24: 156-66. doi: 10.1055/s-00393402746

11. Jurbala BM, Burbank TA. A sonographically guided in-plane distal-to-proximal transligamentous approach to carpal tunnel injections. Hand 2018; 13: 522-28. doi: 10.1177/1558944717725375

12. Grassi W, Farina A, Filippucci E, Cervini C. Intralesional therapy in carpal tunnel syndrome: a sonographic-guided approach. Clin Exp Rheumatol 2002; 20: 73-6. PMID:11892715

13. Smith J, Wisniewski SJ, Finnoff JT, Payne JM. Sonographically guided carpal tunnel injections: the ulnar approach. J Ultrasound Med 2008; 27: 1485-90. doi: $10.7863 /$ jum.2008.27.10.1485

14. Babaei-Ghazani A, Forogh B, Raissi GR, Ahadi T, Eftekharsadat B, Yousefi N, et al. Ultrasound-guided corticosteroid injection in carpal tunnel syndrome: comparison between radial and ulnar approaches. J Pain Res 2020; 13: 1569-78. doi: 10.2147/JPR.S248600

15. Guo XY, Xiong MX, Lu M, Cheng XQ, Wu YY, Chen SY, et al. Ultrasound-guided needle release of the transverse carpal ligament with and without corticosteroid injection for the treatment of carpal tunnel syndrome. J Orthop Surg Res 2018; 13: 69. doi: 10.1186/s13018-018-0771-8

16. Guo K, McCool L, Wang H, Guo D, Guo D. The modified ultrasound-guided distal-to-proximal carpal tunnel injection with median nerve hydrodissection: a retrospective safety review of 827 procedures. Hand 2021; 16: 407-9. doi: $10.1177 / 1558944719861715$

17. Green DP, Mackay BJ, Seiler SJ, Fry MT. Accuracy of carpal tunnel injection: a prospective evaluation of 756 patients. Hand 2020; 15: 54-8. doi: $10.1177 / 1558944718787330$

18. Babaei-Ghazani A, Roomizadeh P, Forogh B, Moeini-Taba SM, Abedini A, Kadkhodaie $M$, et al. Ultrasound-guided versus landmark-guided local corticosteroid injection for carpal tunnel syndrome: a systematic review and meta-analysis of randomized controlled trials. Arch Phys Med Rehabil 2018; 99: 766-75. doi: 10.1016/j.apmr.2017.08.484 
19. Chianca V, Orlandi D, Messina C, Albano D, Corazza A, Rapisarda S, et al Interventional therapeutic procedures to treat degenerative and inflammatory musculoskeletal conditions: state of the art. Radiol Med 2019; 124: 1112-20. doi: 10.1007/s11547-019-01018-8

20. Cartwright MS, White DL, Demar S, Wiesler ER, Sarlikiotis T, Chloros GD, et al. Median nerve changes following steroid injection for carpal tunnel syndrome. Muscle Nerve 2011; 44: 25-9. doi: 10.1002/mus.22067

21. Hsu YC, Yang FC, Hsu HH, Huang GS. Ultrasound-guided corticosteroid injection in patients with carpal tunnel syndrome: efficacy of intra-epineurial injection. Ultraschall Med 2018; 39: 334-42. doi: 10.1055/s-0043-120109

22. Malone DG, Clark TB, Wei N. Ultrasound-guided percutaneous injection, hydrodissection, and fenestration for carpal tunnel syndrome: description of a new technique. J Appl Res 2010; 10: 107-14.

23. Raeissadat SA, Shahraeeni S, Sedighipour L, Vahdatpour B. Randomized controlled trial of local progesterone vs corticosteroid injection for carpal tunnel syndrome. Acta Neurol Scand 2017; 136: 365-71. doi: 10.1111/ ane.12739

24. Lam KHS, Hung CY, Chiang YP, Onishi K, Su DCJ, Clark TB, et al. Ultrasoundguided nerve hydrodissection for pain management: rationale, methods, current literature, and theoretical mechanisms. J Pain Res 2020; 13: 195768. doi: $10.2147 / J P R . S 247208$

25. Uzun H, Bitik O, Uzun Ö, Ersoy US, Aktaş E. Platelet-rich plasma versus corticosteroid injections for carpal tunnel syndrome. J Plast Surg Hand Surg 2017; 51: 301-5. doi: 10.1080/2000656X.2016.1260025

26. Kim DH, Jang JE, Park BK. Anatomical basis of ulnar approach in carpal tunnel injection. Pain Physician 2013; 16: E191-8. PMID: 23703418

27. Lee JY, Park Y, Park KD, Lee JK, Lim OK. Effectiveness of ultrasound-guided carpal tunnel injection using in-plane ulnar approach: a prospective, randomized, single-blinded study. Medicine 2014; 93: e350. doi: 10.1097/ MD.0000000000000350

28. Schrier VJMM, Brault JS, Amadio PC. Ultrasound-guided hydrodissection with corticosteroid injection in the treatment of carpal tunnel syndrome: pilot study. J Ultrasound Med 2020; 39: 1759-68. doi: 10.1002/jum.15279

29. Ustün N, Tok F, Yagz AE, Kizil N, Korkmaz I, Karazincir S, et al. Ultrasoundguided vs. blind steroid injections in carpal tunnel syndrome: a single-blind randomized prospective study. Am J Phys Med Rehabil 2013; 92: 999-1004. doi: 10.1097/PHM.0b013e31829b4d72

30. DeLea SL, Chavez-Chiang NR, Poole JL, Norton HE, Sibbitt WL Jr, Bankhurst AD. Sonographically guided hydrodissection and corticosteroid injection for scleroderma hand. Clin Rheumatol 2011; 30: 805-13. doi: 10.1007/ s10067-010-1653-6

31. Habib GS, Badarny S, Rawashdeh H. A novel approach of local corticosteroid injection for the treatment of carpal tunnel syndrome. Clin Rheumatol 2006 25: 338-40 doi: $10.1007 / \mathrm{s} 10067-005-0002-7$

32. Karimzadeh A, Bagheri S, Raeissadat SA, Bagheri S, Rayegani SM, RahimiDehgolan $S$, et al. The comparison of the effectiveness between different doses of local methylprednisolone injection versus triamcinolone in carpa tunnel syndrome: a double-blind clinical trial. J Pain Res 2019; 12: 579-84. doi: 10.2147/JPR.S190652

33. Salman Roghani R, Holisaz MT, Tarkashvand M, Delbari A, Gohari F, Boon AJ, et al. Different doses of steroid injection in elderly patients with carpal tunnel syndrome: a triple-blind, randomized, controlled trial. Clin Interv Aging 2018; 13: 117-24. doi: 10.2147/CIA.S151290

34. Stark H, Amirfeyz R. Cochrane corner: local corticosteroid injection for carpal tunnel syndrome. J Hand Surg Eur 2013; 38: 911-4. doi: $10.1177 / 1753193413490848$

35. MacMahon PJ, Eustace SJ, Kavanagh EC. Injectable corticosteroid and local anesthetic preparations: a review for radiologists. Radiology 2009; 252: 647 61. doi: $10.1148 /$ radiol.2523081929

36. Li TY, Chen SR, Shen YP, Chang CY, Su YC, Chen LC, et al. Long-term outcome after perineural injection with $5 \%$ dextrose for carpal tunnel syndrome: a retrospective follow-up study. Rheumatology 2021; 60: 881-7. doi: 10.1093/ rheumatology/keaa361

37. Wu YT, Ho TY, Chou YC, Ke MJ, Li TY, Tsai CK, et al. Six-month efficacy of perineural dextrose for carpal tunnel syndrome: a prospective, randomized, double-blind, controlled trial. Mayo Clin Proc 2017; 92: 1179-89. doi: 10.1016/j.mayocp.2017.05.025
38. Catapano M, Catapano J, Borschel G, Alavinia SM, Robinson LR, Mittal N. Effectiveness of platelet-rich plasma injections for nonsurgical management of carpal tunnel syndrome: a systematic review and meta-analysis of randomized controlled trials. Arch Phys Med Rehabil 2020; 101: 897-906. doi: 10.1016/j.apmr.2019.10.193

39. Bahrami MH, Shahraeeni S, Raeissadat SA. Comparison between the effects of progesterone versus corticosteroid local injections in mild and moderate carpal tunnel syndrome: a randomized clinical trial. BMC Musculoskelet Disord 2015; 16: 322. doi: 10.1186/s12891-015-0752-6

40. Wu YT, Chen SR, Li TY, Ho TY, Shen YP, Tsai CK, et al. Nerve hydrodissection for carpal tunnel syndrome: a prospective, randomized, double-blind, controlled trial. Muscle Nerve 2019; 59: 174-80. doi: 10.1002/mus.26358

41. Evers S, Thoreson AR, Smith J, Zhao C, Geske JR, Amadio PC. Ultrasoundguided hydrodissection decreases the gliding resistance of the median nerve within the carpal tunnel. Muscle Nerve 2018; 57: 25-32. doi: 10.1002/ mus. 25723

42. Evers $S$, Bryan AJ, Sanders TL, Gunderson $T$, Gelfman R, Amadio PC Influence of injection volume on rate of subsequent intervention in carpal tunnel syndrome over 1-year follow-up. J Hand Surg Am 2018; 43: 537-44. doi: 10.1016/j.jhsa.2018.02.024

43. Lin $\mathrm{MT}$, Liao $\mathrm{CL}$, Hsiao MY, Hsueh $\mathrm{HW}$, Chao $\mathrm{CC}, \mathrm{Wu} \mathrm{CH}$. Volume matter in ultrasound-guided perineural dextrose injection for carpal tunnel syndrome: a randomized, double-blinded, three-arm trial. Front Pharmacol 2020; 11: 625830. doi: 10.3389/fphar.2020.625830

44. Armstrong T, Devor W, Borschel L, Contreras R. Intracarpal steroid injection is safe and effective for short-term management of carpal tunnel syndrome. Muscle Nerve 2004; 29: 82-8. doi: 10.1002/mus.10512

45. Atroshi I, Flondell M, Hofer M, Ranstam J. Methylprednisolone injections for the carpal tunnel syndrome: a randomized, placebo-controlled trial. Ann Intern Med 2013; 159: 309-17. doi: 10.7326/0003-4819-159-5-20130903000004

46. Cass SP. Ultrasound-guided nerve hydrodissection. Current Sports Medicine Reports 2016; 15: 20-2. doi: 10.1249/jsr.0000000000000226

47. Karadaş Ö, Tok F, Akarsu S, Tekin L, Balaban B. Triamcinolone acetonide vs procaine hydrochloride injection in the management of carpal tunne syndrome: a randomized placebo-controlled study. J Rehabil Med 2012; 44: 601-4. doi: 10.2340/16501977-0990

48. Elawamy A, Hassanien M, Hamed A, Roushdy ASI, Abass NA, Mohammed $\mathrm{G}$, et al. Efficacy of hyalase hydrodissection in the treatment of carpal tunnel syndrome: a randomized, double-blind, controlled, clinical trial. Pain Physician 2020; 23: E175-83. PMID: 32214296

49. Nwawka OK, Miller TT, Jawetz ST, Saboeiro GR. Ultrasound-guided perineural injection for nerve blockade: does a single-sided injection produce circumferential nerve coverage? J Clin Ultrasound 2016; 44: 465-9. doi: 10.1002/jcu.22364

50. Chen LC, Ho TY, Shen YP, Su YC, Li TY, Tsai CK, et al. Perineural dextrose and corticosteroid injections for ulnar neuropathy at the elbow: a randomized double-blind trial. Arch Phys Med Rehabil 2020; 101: 1296-303. doi 10.1016/j.apmr.2020.03.016 